\title{
Optimum Determination of Partial Transmission Ratios of Three-Step Helical Gearboxes for Getting Minimum Cross Section Dimension
}

\author{
Vu Ngoc Pi and Nguyen Khac Tuan \\ Thai Nguyen University of Technology, Thai Nguyen 23000, Vietnam
}

\begin{abstract}
This paper presents a new study on optimum calculation of partial ratios of three-step helical gearboxes. The chosen objective function is the cross section dimension of the gearbox. In solving the optimization problem, the design equation for pitting resistance of a gear set was investigated and equations on moment equilibrium condition of a mechanic system including three gear units and their regular resistance condition are analyses. From the results of the study, effective formula for determination of the partial ratios of three-step helical gearboxes is introduced. As the formulas are explicit, the partial ratios can be calculated accurately and simply.
\end{abstract}

Key words: Gearbox design, optimum design, helical gearbox, transmission ratio.

\section{Introduction}

In optimum design of gearboxes, the determination of the partial transmission ratios has a decisive role. This is because the size, the mass, and also the cost of the gearboxes depend strongly on the partial ratios. Therefore, optimum determination of the partial ratios has been subjected to many researches.

Up to now, many researches have been carried out on the prediction of the partial ratios of the three-step helical gearboxes. Kudreavtev et al. [1] introduced a graph method (Fig.1) for determining the partial ratios of the first and the second steps for minimal mass of the gearbox. The transmission ratio of the third step is then calculated from the total ratio and the ratios of the first and the second steps. The graph method was also presented in other study of Petrovski et al. [2] in order to find the partial ratios for minimal volume of gears. Romhild et al. [3] gave models for calculation of the partial ratios for minimal mass of the gearbox. Recently, Ngoc et al. [4] proposed models which are used to determine the partial ratios for the minimum

Corresponding author: $\mathrm{Vu}$ Ngoc $\mathrm{Pi}$, Ph.D., associate professor, main research fields: optimum design of machine elements, abrasive machining. mass or the gears. Also, Ngoc [5] carried out a study on the calculation of the partial ratios of three-step helical gearboxes with the first and third-step double gear-set in order to get the minimum mass of gears.

It is clear that there have been many studies on splitting the total transmission ratio for three-step helical gearboxes. Nevertheless, the optimum partial ratios for getting the minimum cross section dimension of the gearbox, a very important objective, has not been investigated. In this paper, a new result for optimum calculation of partial ratios for three-step helical gearboxes for getting the minimum cross section dimension is introduced.

\section{Optimal Calculation of Partial Transmission Ratio}

For the first step of the gearbox, Eq. (1) is used as the design equation for pitting resistance [6]:

$$
\sigma_{H 1}=Z_{M 1} \cdot Z_{H 1} \cdot Z_{c 1} \cdot \sqrt{\frac{2 \cdot T_{11} \cdot K_{H 1} \cdot \sqrt{u_{1}+1}}{b_{w 1} \cdot d_{w 11}^{2} \cdot u_{1}}} \leq\left[\sigma_{H 1}\right]
$$

It follows from Eq. (1) that:

$$
\left[T_{11}\right]=\frac{b_{w 1} \cdot d_{w 11}^{2} \cdot u_{1}}{2 \cdot\left(u_{1}+1\right)} \frac{\left[\sigma_{H 1}\right]^{2}}{K_{H 1} \cdot\left(Z_{M 1} \cdot Z_{H 1} \cdot Z_{\varepsilon 1}\right)^{2}}
$$




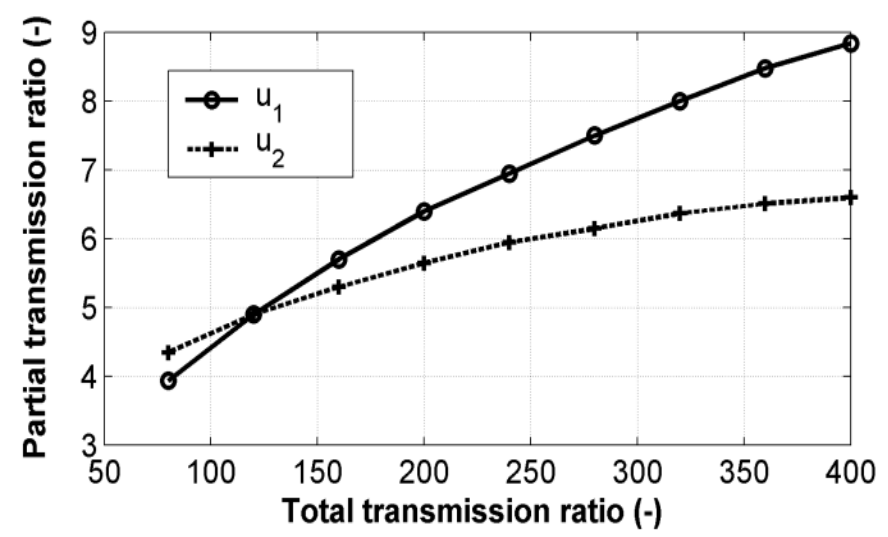

Fig. 1 Partial ratios of steps 1 and 2 versus the total transmission ratio [1].

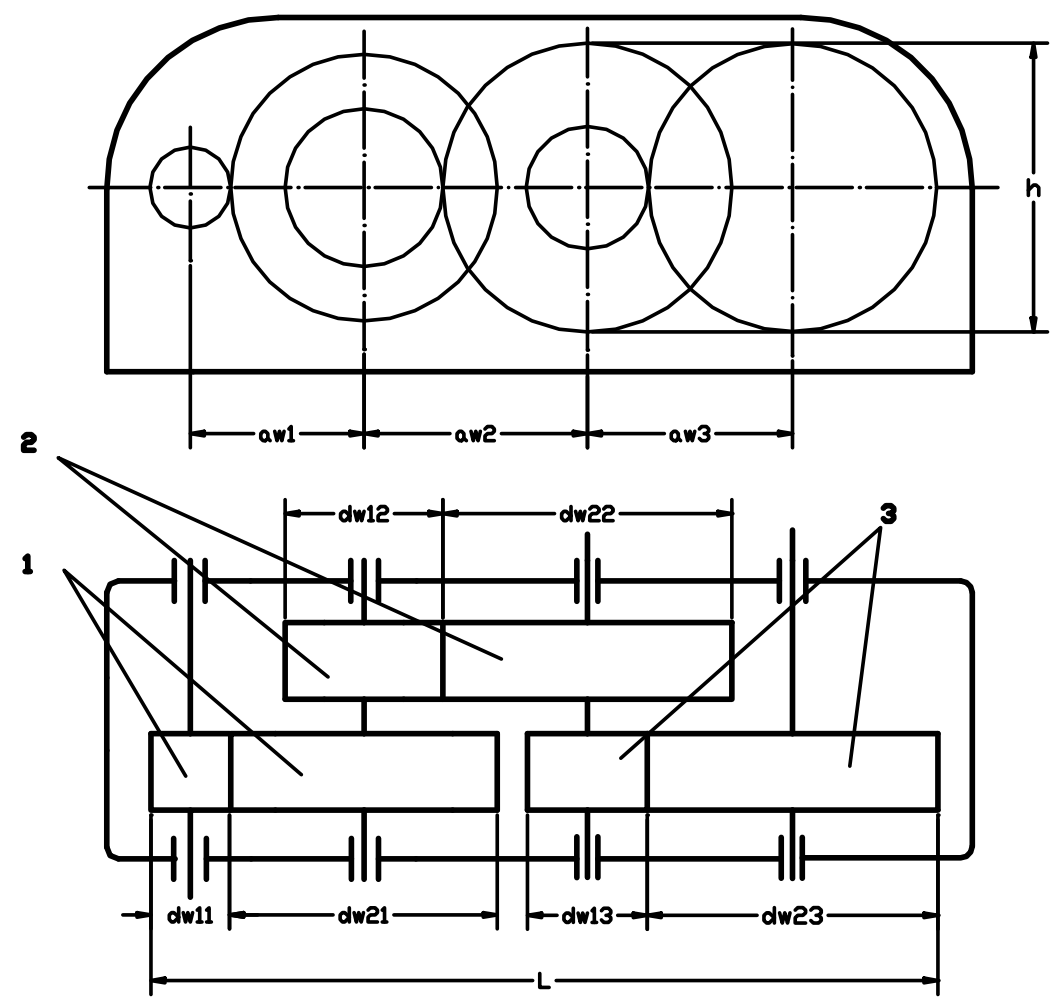

Fig. 2 Calculating schema for three-step helical gearbox.

where, $b_{w 1}$ is the face width $(\mathrm{mm})$ and $d_{w 11}$ is the pitch diameter of the first step; $b_{w 1}$ and $d_{w 11}$ are calculated by Eqs. (3)-(4):

$$
\begin{gathered}
b_{w 1}=\psi_{b a 1} \cdot a_{w 1}=\psi_{b a 1} \cdot d_{w 11} \cdot\left(u_{1}+1\right) / 2 \\
d_{w 11}=d_{w 21} / u_{1}
\end{gathered}
$$

Substituting Eqs. (3) and (4) into Eq. (2), authors get

$$
\left[T_{11}\right]=\frac{\psi_{b a 1} \cdot d_{w 21}^{3} \cdot\left[K_{01}\right]}{4 \cdot u_{1}^{2}}
$$

in which

$$
\left[K_{01}\right]=\frac{\left[\sigma_{H 1}\right]^{2}}{K_{H 1} \cdot\left(Z_{M 1} \cdot Z_{H 1} \cdot Z_{\varepsilon 1}\right)^{2}}
$$

From Eq. (5), the pitch diameter $d_{w 21}$ can be calculated by 


$$
d_{w 21}=\left(\frac{4\left[T_{11}\right] u_{1}^{2}}{\psi_{b a 1}\left[K_{01}\right]}\right)^{1 / 3}
$$

Calculating in the same manner, Eqs. (8)-(9) are found for the second and the third steps:

$$
\begin{aligned}
& d_{w 22}=\left(\frac{4\left[T_{12}\right] u_{2}^{2}}{\psi_{b a 2}\left[K_{02}\right]}\right)^{1 / 3} \\
& d_{w 23}=\left(\frac{4\left[T_{13}\right] u_{3}^{2}}{\psi_{b a 3}\left[K_{03}\right]}\right)^{1 / 3}
\end{aligned}
$$

For a three-step helical gearbox (Fig. 2), the cross-sectional dimension is minimum when [1]:

$$
d_{w 21}=d_{w 22}=d_{w 23}
$$

From Eqs. (7), (8) and (10), Eq. (11) can be written

$$
\frac{\left[T_{11}\right] u_{1}^{2}}{0.25 \psi_{b a 1}\left[K_{01}\right]}=\frac{\left[T_{12}\right] u_{2}^{2}}{0.25 \psi_{b a 2}\left[K_{02}\right]}
$$

with

$$
\left[T_{12}\right]=\left[T_{11}\right] \cdot u_{1} \cdot \eta_{b r} \cdot \eta_{o}
$$

where, $\left[T_{12}\right]$ is permissible torque on the drive shaft of the second step (Nmm); $\eta_{b r t}$ is helical gear transmission efficiency ( $\eta_{b r t}$ is from 0.96 to 0.98 [6]); $\eta_{o}$ is transmission efficiency of a pair of rolling bearing ( $\eta_{o}$ is from 0.99 to 0.995 [6]). Choosing $\eta_{b r t}=0.97, \eta_{o}=0.992$ and substituting Eq. (12) into Eq. (11) gives:

$$
u_{1}=\frac{0.96}{c_{k 1} c_{b a 1}} u_{2}^{2}
$$

with $c_{k 1}=\left[k_{02}\right] /\left[k_{01}\right], c_{b a 1}=\psi_{b a 2} / \psi_{b a 1}$.

In exactly similar manner, the transmission ratio of the second step is calculated as:

$$
u_{2}=\frac{0.96}{c_{k 2} c_{b a 2}} u_{3}^{2}
$$

with $c_{k 2}=\left[k_{03}\right] /\left[k_{02}\right], c_{b a 2}=\psi_{b a 3} / \psi_{b a 2}$.

In practice $c_{b a i}=1.2 \div 1.3 ; c_{k i} \approx 1 \div 1.1$ (with $\mathrm{i}$ $=1$; 2; 3) [6]; Choosing $c_{b a i}=1.2 ; c_{k i}=1$ and substituting them into Eqs. (13) and (14) gives

$$
\begin{aligned}
& u_{1}=0.8 u_{2}^{2} \\
& u_{2}=0.8 u_{3}^{2}
\end{aligned}
$$

Also, the total transmission ratio of the gearbox is determined by:

$$
u_{h}=u_{1} \cdot u_{2} \cdot u_{3}
$$

Solving Eqs. (15), (16) and (17) gets

$$
\begin{aligned}
& u_{1}=0.8527 \cdot u_{h}^{0.5714} \\
& u_{2}=1.0324 \cdot u_{h}^{0.2857} \\
& u_{3}=1.136 \cdot u_{h}^{0.1429}
\end{aligned}
$$

\section{Conclusion}

The minimal cross section dimension of a three-step helical gearbox can be obtained by optimum splitting the total transmission ratio of the gearbox.

Models for determination of the optimum partial ratios for getting the minimum cross section dimension of the gearbox are proposed.

By using explicit models, the partial ratios of the gearbox can be calculated accurately and simply.

\section{References}

[1] Kudreavtev, V. N., Gierzaves. I. A., and Glukharev, E. G. 1971. Design and Calculus of Gearboxes. Sankt Petersburg: Mashinostroenie Publishing.

[2] Petrovski, A. N., Sapiro, B. A., and Saphonova, N. K. 1987. "About Optimal Problem for Multi-step Gearboxes." Vestnik Mashinostroenie 10: 13-4.

[3] Romhild, I., and Linke, H. 1992. "Gezielte Auslegung Von Zahnradgetrieben mit minimaler Masse auf der Basis neuer Berechnungsverfahren." Konstruktion 44 (7-8): 229- 36.

[4] Pi, V. N., Binh, N. D., and Dac, V. Q. 2006. "Phan Quang The, Optimal Calculation of Total Transmission Ratio of Three-Step Helical Gearboxes for Minimum Mass of Gears." Journal of Science and Technology of 6 Engineering Universities 55:91-3.

[5] Ngoc Pi, V. 2008. "Optimum Determination of Partial 

Minimum Cross Section Dimension

Transmission Ratios of Three-Step Helical Gearboxes with First and Third-Step Double Gear-Set for Minimal Mass of Gears." In American Conference on Applied Mathematics (Math's 08), Harvard, Massachusetts, USA,
March 24-26, 385-8.

[6] Chat, T., and Le Van U. 1998. Design and Calculus of Mechanical Transmissions, Hanoi, Educational Publishing House. 